\title{
Cerebellar Guidance of Premotor Network Development and Sensorimotor Learning
}

\author{
Sherwin E. Hua and James C. Houk ${ }^{1}$ \\ Department of Physiology \\ Northwestern University Medical School \\ Chicago, Illinois 60611-3008
}

\begin{abstract}
Single unit and imaging studies have shown that the cerebellum is especially active during the acquisition phase of certain motor and cognitive tasks. These data are consistent with the hypothesis that particular sensorimotor procedures are acquired and stored in the cerebellar cortex and that this knowledge can then be exported to the cerebral cortex and premotor networks for more efficient execution. In this article we present a model to illustrate how the cerebellar cortex might guide the development of cortical-cerebellar network connections and how a similar mechanism operating in the adult could mediate the exportation of sensorimotor knowledge from the cerebellum to the motor cortex. The model consists of a three-layered recurrent network representing the cerebello-thalamocorticalponto-cerebellar limb premotor network. The cerebellar cortex is not explicitly modeled. Our simulations show that Hebbian learning combined with weight normalization allows the emergence of reciprocal and modular structure in the limb premotor network. Reciprocal connections allow activity to reverberate around specific loops. Modularity organizes the connections into specific channels. Furthermore, we show that cerebellar learning can be exported to motor cortex through these modular and reciprocal premotor circuits. In particular, we simulate developmental alignment of visuomotor relations and their realignment as a consequence of prism exposure. The
\end{abstract}

\footnotetext{
${ }^{1}$ Corresponding author.
}

exportation of sensorimotor knowledge from the cerebellum to the motor cortex may allow faster and more efficient execution of learned motor responses.

\section{Introduction}

It is now well established that the cerebellum plays an important role in sensorimotor learning (Thompson 1986; Ito 1989; Thach et al. 1992; Houk et al. 1996; Raymond et al. 1996). Although cerebellar patients are able to make a wide range of movements, their ability to adapt to challenging conditions is severely impaired (Thach et al. 1992). In addition to basic movement skills, imaging studies show that the cerebellum is involved in complex sensorimotor functions that include cognitive components (Kim et al. 1994; Raichle et al. 1994; Friston et al. 1992).

Although the parallel fiber-Purkinje cell (PC) synapse is undoubtedly one important site at which learning takes place (Marr 1969; Albus 1971; Ito 1989; Berthier et al. 1993), learning mechanisms are widespread, and sensorimotor learning is undoubtedly a distributed function (Houk and Barto 1992; Bloedel et al. 1996). The storage capacity of the cerebellar cortex is quite enormous (Gilbert 1974; Tyrrell and Willshaw 1992), so one possibility is that a diversity of complex sensorimotor memories are stored in the cerebellum and that during practice, these programs are exported to premotor networks for more efficient execution (Galiana 1986; Houk and Barto 1992). Consistent with this hypothesis, single unit activity in the cerebellum is particularly intense during the acquisition phase of conditioned forelimb movements (Milak et al. 1995). Similarly, imaging studies indicate the most intense cerebellar activations when sensorimotor tasks are being learned (Friston et al. 1992; Ebner et al. 1996). Cognitive functions of the cerebellum could similarly be exported to the cerebral cortex to improve

LEARNING \& MEMORY 4:63-76 @ 1997 by Cold Spring Harbor Laboratory Press ISSN1072-0502/97 \$5.00

$$
\begin{array}{lllllllllllllllll} 
& E & A & R & N & I & N & G & \mathbf{Q} & M & E & M & O & R & Y \\
63 & &
\end{array}
$$


the efficiency of thinking (Houk 1997). Positron emission tomography (PET) studies showed that metabolic activity associated with a cognitive verbfinding task moved from the lateral cerebellum to a cortical site with practice (Raichle et al. 1994). These results, along with previous studies showing that cerebellar damage hinders normal motor learning but not the retention of motor memories, suggest that the cerebellar cortex can export learning of motor and cognitive tasks to premotor and cortical sites.

Although cerebellar learning has been modeled extensively, mechanisms whereby cerebellar knowledge might be exported to premotor and cortical sites have not received much attention. Here we explore this issue with respect to limb movement control. The premotor network that controls voluntary movements of the limb consists of a cerebello-thalamo-cortico-ponto-cerebellar circuit regulating motor cortical output and a cerebello-rubro-reticulo-cerebellar brain stem circuit regulating red nucleus output (for review, see Houk et al. 1993). Experiments have shown that the brain stem circuit exhibits reverberatory activity generated by recurrent positive feedback in the network (Tsukahara et al. 1983; Keifer et al. 1992; Keifer 1996). Workers using modeling efforts have assumed that the limb premotor network is comprised of reciprocal and topographically specific connections that segregate the control of movement into computational modules, each of which generates an elemental motor command (Houk et al. 1990; Berthier et al. 1993). Such specificity in the premotor circuits may allow activity patterns to be readily shaped by inhibitory output from cerebellar PCs to coordinate populations of motor cortical and red nucleus commands (Houk et al. 1993). Developmental plasticity leading to the formation of the postulated premotor modules might represent a neonatal phase of the adult plasticity involved in the exportation process.

In addition to the formation of the premotor network, developmental alignment of premotor circuits with cortico-cortical connections may guide the organization of sensorimotor maps in the cortex. For example, monkeys deprived of sight of their hands and body during development showed difficulty making and adapting visually guided movements later in life (Bauer and Held 1971). Thus, the establishment of these visuo-motor maps during development may be important for normal visuo-motor adaptation guided by cerebellar and premotor circuits later in life.
In this study we provide a computational model that first simulates the development of specific reciprocal circuits in the premotor network. Later in development, these reciprocal circuits guide the alignment of visuo-motor maps in cortico-cortical connections. After these two developmental stages are completed, the model simulates the exportation of cerebellar learning to corticocortical networks for a visuo-motor adaptation task analogous to prism adaptation of limb movements. To simplify the model, cerebellar learning is not explicitly modeled. Previous modeling efforts have dealt with cerebellar learning of visuomotor relations (Berthier et al. 1993). Instead, we focus on plasticity in premotor and cortical networks that is guided by the cerebellum.

The centerpiece of the model is the cerebellothalamo-cortico-ponto-cerebellar recurrent network. Because the thalamo-cortical circuit is itself a recurrent network within the larger recurrent premotor network, we simplify the network by incorporating the recurrent thalamo-cortical circuit into a single layer of units having lateral connections and self-connections. The self-connections on this layer represent specific thalamo-cortical reciprocal loops that allow cortical activity to persist through thalamic connections. Lateral connections represent intrinsic cortical connectivity as well as limited divergence of thalamo-cortical recurrent connections. The incorporation of the thalamo-cortical circuit into a single layer is justified by experimental evidence demonstrating topographic specificity in reciprocal thalamo-cortical connections (Ma and Juliano 1991; Ghosh et al. 1994). Additionally, we have previously demonstrated that any two-layered recurrent network will develop reciprocal and symmetric connections through Hebbian learning (S.E. Hua, F.A. Mussa-Ivaldi, and J.C. Houk, unpubl.). The reciprocal connections in such twolayered networks can be approximated by a single composite weight matrix constructed by the product of the feed-forward and feedback weight matrices in the network. For topographically organized reciprocal networks, the composite weight matrix has a banded diagonal structure, which is equivalent to having self-weights and lateral connections.

Our simulations show that Hebbian learning during the early developmental stage allows the formation of reciprocal and modular connections in the premotor network. Reciprocity exists when activity initiated in a unit will return to that unit after traveling through multisynaptic connections

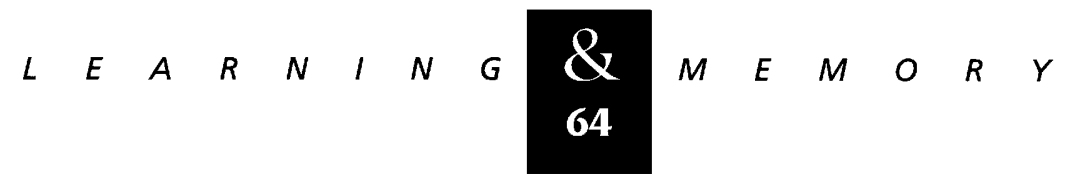


in the network (self-connections are reciprocal but are not considered in this study). Modularity exists when a group of cells in the network has stronger connections with cells in the same group than with cells in other groups. We also define computational or graded modularity as a subclass of modularity in which cells have a uniformly graded distribution of connections that varies with functional or topographic similarity. For example, cortical units that have stronger connections with units having similar directional tuning or orientation selectivity have graded modularity.

Development of the premotor network is guided by spontaneous PC disinhibition of neighboring nuclear cells. Once development of the premotor network has occurred, the cerebellum is able to direct the alignment of visuo-motor maps in the cortex. Furthermore, the cerebellum can direct learning and realignment of cortico-cortical circuits by exporting cerebellar learning to cortical sites. After learning, cortico-cortical connections in our model are able to initiate movements in the correct direction in a visuo-motor adaptation task without direct control by the cerebellum.

\section{Materials and Methods}

The model premotor network is comprised of a three-layered recurrent network representing the cerebellar nucleus, the motor cortical-thalamic circuit, and the pontine nucleus, as schematized in Figure 1 (CBN, MC, and PN, respectively). A fourth layer representing cortical regions that specify visual target information (sensory cortex) projects to the motor cortex. These cortico-cortical connections are assumed to develop after connections in the premotor network have organized. Each layer of units is wrapped, meaning the ends were connected, to reduce border effects. Thus, effectively, each layer is circular in topology.

In the cerebellar nucleus layer, we assume for simplicity that each PC inhibits a cluster of three neighboring nuclear cells. This topographic arrangement allows better visualization of the effects of correlated PC disinhibition through the topography of resulting premotor connections. During the development of the premotor loop, nuclear cells have spontaneous activity, such that when inhibition from a PC is removed, the cluster of nuclear cells that received its inhibition becomes active. Later, after the premotor network has developed, we assume that cerebellar nuclear cells

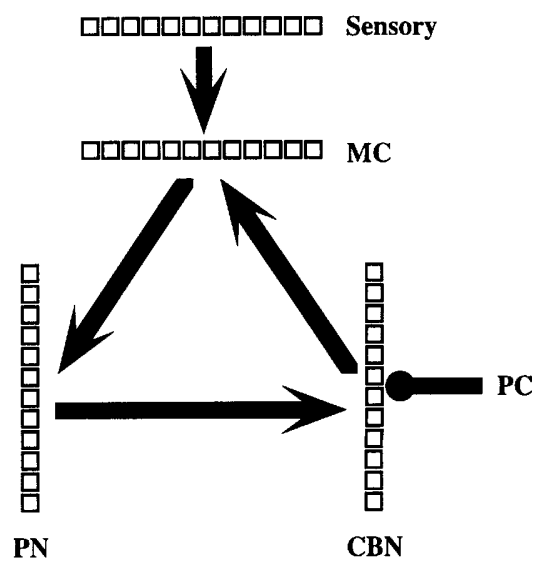

Figure 1: Schematic diagram of the network. The three-layered premotor network is comprised of the motor cortico-thalamo-cortical (MC) layer, the pontine (PN) layer, and the cerebellar nuclear (CBN) layer. Adaptable cortico-cortical connections exist between the sensory layer and the MC layer. PC inhibition regulates the CBN layer.

are no longer spontaneously active. Instead, excitatory pontine inputs are necessary in addition to PC disinhibition to activate nuclear cells. No inhibitory interneurons are included in the cerebellar nuclear layer.

Cerebellar nuclear activation is transmitted to the motor cortex via thalamic connections. For simplicity the reciprocal cortical-thalamic circuit is represented by a single layer of units (MC layer) with lateral excitatory connections and self connections. Self-connections represent reciprocal cortico-thalamic connections, whereas lateral connections represent both the intrinsic cortical connections as well as divergent cortico-thalamo-cortical connections. Additionally, inhibitory interneurons are included to stabilize cortical activity.

The pontine layer is a relay layer without external inputs other than the MC layer and without intrinsic lateral excitatory connections. Inhibitory interneurons are included on this layer. Histological studies have demonstrated the existence of principal relay cells that project to the cerebellum as well as inhibitory interneurons in the pontine nucleus (Brodal and Bjaalie 1992).

We assume that cortico-cortical connections develop after the basic organization of the premotor loop has been laid down. After this point, we fix the connections in the premotor loop and allow cerebellar learning to direct the alignment and realignment of the feed-forward connections from the sensory layer to the motor cortex. The sensory

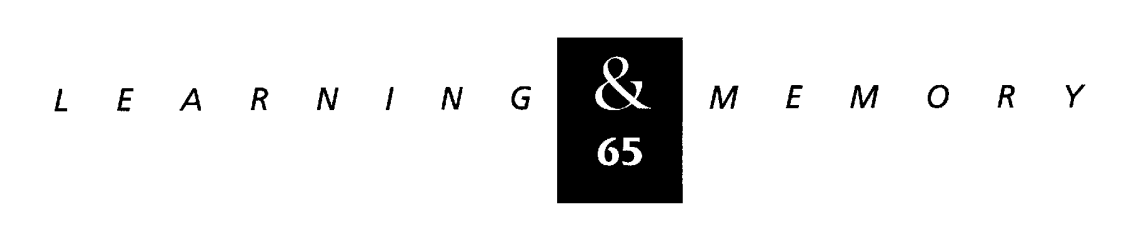


layer represents cortical regions such as parietal cortex or ventral premotor cortex that provide information about visual targets to which movements are to be made. The spatially correlated external inputs representing visual target information are implemented in the model as a cluster of three neighboring units on the sensory layer. Additionally, a corresponding PC is turned off resulting in the disinhibition of three neighboring cerebellar nuclear cells. The concurrent activation of premotor cortical units and disinhibition of cerebellar nuclear cells drives the formation of an appropriate cortico-cortical mapping.

Two dynamics occur simultaneously in the network, namely activation dynamics and synaptic efficacy or learning dynamics (S.E. Hua, F.A. MussaIvaldi, and J.C. Houk, in prep.). The activation states of all units are updated asynchronously and are governed by the additive model,

$$
\begin{aligned}
& x_{j}(t+d t)= \\
& x_{j}(t)+\left[-\frac{1}{R} x_{j}(t)+\sum_{i} W_{j i}(t) \sigma\left[x_{i}(t)\right]+b_{j}(t)\right] \frac{d t}{C}
\end{aligned}
$$

where $x_{j}(t)$ represents the instaneous activation state $\sigma\left[x_{j}(t)\right]$ is the activation function whose value is comparable to firing rate, $W_{j i}(t)$ is the synaptic weight from unit $i$ to unit $j, R C$ is the time constant for activation dynamics, and $b_{j}(t)$ represents the time-varying inputs.

The activation rule used in the simulations is piecewise and is similar to Anderson's brain-statein-a-box model (Anderson et al. 1977):

$$
\sigma(x)=\left\{\begin{array}{cc}
1 & \text { if } x \geqslant 1 \\
x & \text { if } 0<x<1 \\
0 & \text { if } x \leqslant 0
\end{array}\right\}
$$

Learning occurs by $d W_{j i}(t)=\frac{\alpha}{\tau_{w}} \sigma\left[x_{j}(t)\right] \sigma\left[x_{i}(t)\right] d t$, the general Hebbian update rule where $\alpha$ is the learning rate and $\tau_{w}$ is the time constant for continuous learning. Becuase Hebbian learning in this form is generally unstable (Miller and MacKay 1994), we impose both presynaptic and postsynaptic normalization as a means of stabilizing Hebbian learning.

$$
W_{j i}(t+d t)=\beta\left[W_{j i}(t)\right]_{\mathrm{post}}\left[W_{j i}(t)+d W_{j i}(t)\right] \beta\left[W_{j i}(t)\right]_{\mathrm{pre}}
$$

where the coefficient for presynaptic normalization is defined by

$$
\beta\left[W_{j i}(t)\right]_{\mathrm{pre}}=\frac{X_{\mathrm{pre}}}{\sum_{b}\left[W_{b i}(t)+d W_{b i}(t)\right]}
$$

and the coefficient for postsynaptic normalization is defined by

$$
\beta\left[W_{j i}(t)\right]_{\mathrm{post}}=\frac{\chi_{\text {post }}}{\sum_{b}\left[W_{j h}(t)+d W_{j h}(t)\right]}
$$

$X_{\text {pre }}$ is the limit on the sum of all weights from each presynaptic unit, and $X_{\text {post }}$ is the limit on the sum of all weights onto each postsynaptic unit. For both normalization conditions to be satisfied, the condition $X_{\text {pre }}=X_{\text {post }}$ must hold. (For presynaptic normalization, we have $\sum_{j=1}^{N} W_{j i}=\chi_{\text {pre }}$. For postsynaptic normalization we have $\sum_{i=1}^{N} W_{j i}=\chi_{\text {post }}$. Because $\sum_{i=1}^{N} \sum_{j=1}^{N} W_{j i}=N \chi_{\text {pre }}$ and $\sum_{j=1}^{N} \sum_{i=1}^{N} W_{j i}=$ $N \chi_{\text {post }}$, it follows that $\chi_{\text {post }}=\chi_{\text {pre }}$ must hold if both normalization conditions are to be satisfied.) $\mathrm{Al}$ though both learning and activation dynamics evolve simultaneously, the dynamics for weight update are slower than that for changes in the instantaneous firing rate, that is, $R C<<\tau_{w}$. Typically, $R C$ $=1, \tau_{w}=1000, d t=0.1$, and $\alpha=1$.

In addition to the excitatory projection neurons described above, linear inhibitory interneurons are included in the cortical and pontine layers. Each interneuron inhibits only one projection neuron with a weight of -1 and receives excitatory input from all projection units on the previous layer with a weight equal in magnitude to the mean of all excitatory weights of projection units. We assume that inhibitory interneurons have fast dynamics such that the time delay through the interneurons is negligible compared with the direct excitatory connections. Finally, noise is added to the weights at each time step at $\pm 10 \%$ of the value of the largest weight. This noise represents spontaneous synaptic sprouting and elimination.

\section{Results}

The influence of cerebellar output on the learning and development of downstream circuits is simulated in three stages. In the first stage, initially random connections in the recurrent premotor network are organized into specific reciprocal 
circuits. Cerebellar activation during this developmental stage allows the alignment of specific PCs with specific reciprocal channels in the premotor circuit. In the second stage, premotor circuits are assumed to have passed the critical period of development and are no longer plastic. Visuo-motor maps in cortico-cortical projections are aligned by simultaneous presentation of visual target input to both the sensory layer and the cerebellar cortex. We assume that the cerebellar cortex has already learned how to command movements to these visual targets. In the third stage, cerebellar knowledge about prism distortion is exported to corticocortical connections through activation of specific premotor pathways.

\section{DEVELOPMENT OF THE PREMOTOR CIRCUIT}

The feedback circuit consists of a cerebellar nuclear layer, a cortical-thalamic layer, and a pontine layer as schematized in Figure 1. Each of the three projections in this circuit was initialized with random connections. The only external input to the network at this stage was PC inhibition of nuclear units. During development, we assumed that PCs are spontaneously active with intermittent periods of inactivity. We simulated intermittent PC inactivity by activating neighboring clusters of cerebellar nuclear cells for 75 time steps, a conservative number of steps to ensure network settling.
Under these developmental conditions, the connections in the feedback circuit developed reciprocal connections, as shown in Figure 2. Figure 2 , a-c, shows the weight matrices for the cerebello-MC, MC-pontine, and ponto-cerebellar projections, respectively. Figure $2, \mathrm{~d}-\mathrm{f}$, shows the corresponding composite matrices for the cerebellar, MC, and pontine layers, respectively. Each composite matrix shows the effective connections that a layer makes with itself through the feedback connections in the network and is calculated by the product of the three individual weight matrices, taken in reverse order. The diagonal terms of the composite matrix represent the effective connection that a single unit makes with itself through the network; thus the diagonal terms give a measure of the degree of reciprocity in the network.

Figure $2 \mathrm{a}$ shows that the cerebello-MC projection developed a smooth topographic map. The $\mathrm{MC}$-pontine and ponto-cerebellar projections developed partial topographic maps. The MC-pontine projection, shown in Figure $2 \mathrm{~b}$, has topographic receptive fields but discontinuous projection fields, such that neighboring MC units (labeled as layer 2) project to similar, topographically discontinuous pontine units. On the other hand, the ponto-cerebellar projection, shown in Figure 2c, has topographic projection fields but discontinuous receptive fields, such that neighboring cerebellar units have similar but discontinuous receptive fields.
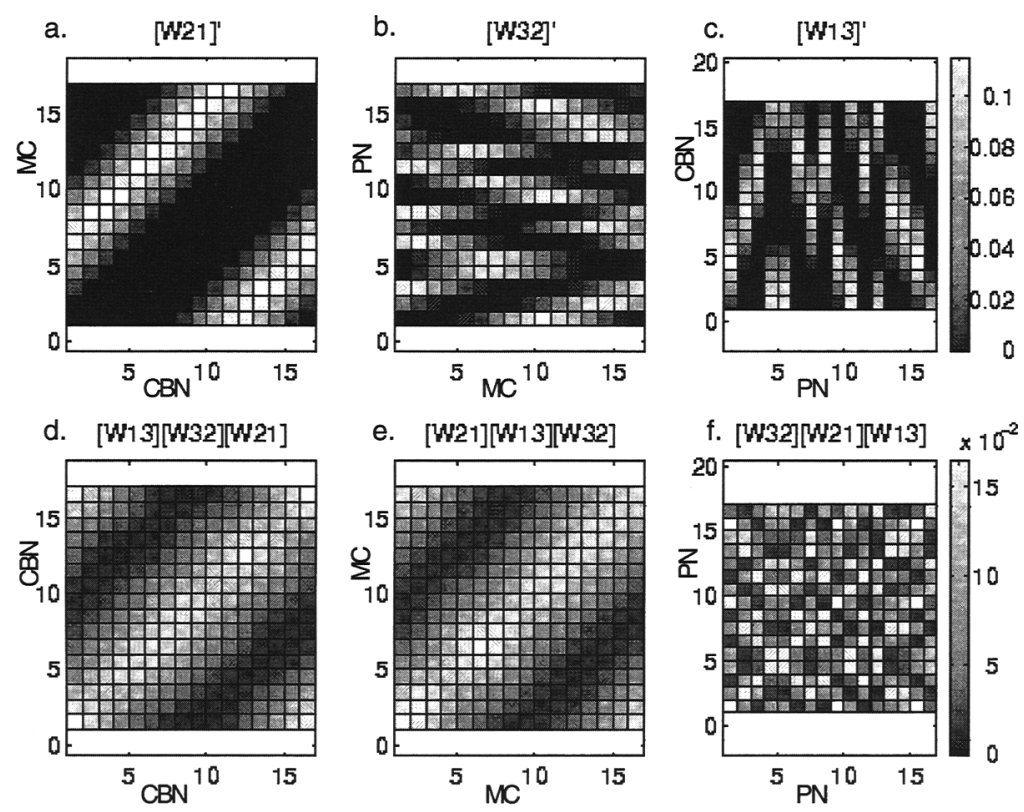

Figure 2: Weight matrices $(a-c)$ and composite matrices $(d-f)$ of the premotor network after the first developmental phase of Hebbian learning. (a) The weight matrix $W_{21}$ from the cerebellar layer (layer 1) to the MC layer (layer 2); (b) the weight matrix $W_{32}$ from the $M C$ layer to the pontine layer (layer 3); (c) the weight matrix $W_{13}$ from the pontine layer to the cerebellar layer; $(d)$ the composite matrix for the cerebellar layer, constructed by the product of the three weight matrices $\mathrm{W}_{13} \mathrm{~W}_{32} \mathrm{~W}_{21}$; (e) the composite matrix for the MC layer, i.e., $W_{21} W_{13} W_{32} ;(f)$ the composite matrix for the pontine layer, i.e., $W_{32} W_{21} W_{13}$. 
These weight maps are consistent with the correlation structure between individual units on each of the three layers. Both the MC and cerebellar layers have topographically correlated activity. Correlation of MC units arises from lateral connections between neighboring units. On the other hand, correlation of cerebellar nuclear cells is generated by correlated disinhibition by each PC. Units on the pontine layer do not have any correlation in activity imposed by the network architecture. Thus, the cerebello-MC projection between two layers with correlated activity develops a fully topographic map. The MC-pontine projection from a layer with correlated activity to a layer without correlations develops topographic receptive fields but nontopographic projection fields. The ponto-cerebellar projection from a layer without correlations to a layer with correlated activity develops topographic projection fields but non-topographic receptive fields.

As defined above, the composite matrix shows the multisynaptic connections that a unit makes with all other units on the same layer. The composite matrix for the cerebellar layer (Fig. 2d) is banded diagonal. This matrix structure shows that activity initiated in a cerebellar unit will travel through the other two layers and will return not only to that unit, by reciprocal connections as illustrated by the diagonal terms, but will also travel to neighboring cerebellar units, as exemplified by off-diagonal terms. The composite matrix for the MC, shown in Figure $2 \mathrm{e}$, has a similar banded diagonal structure. In contrast, the composite matrix for the pontine nucleus, shown in Figure $2 \mathrm{f}$, is a symmetric but otherwise disorganized matrix with prominent diagonal terms.

Once again, these composite matrices are consistent with the correlation structure between individual units on each layer. First, all three composite matrices have prominent diagonal terms. Diagonal terms on a composite matrix represent reciprocal connections that allow activity to travel through the network and return to the units from which the activity originated. Second, all three composite matrices are symmetric. Symmetry shows that the influence of unit A on unit $B$ is the same as that of unit B on unit A through multisynaptic connections in the network. Finally, for layers with correlated activity, the composite matrix is banded, representing a propensity for recurrent activity to return not only to units that initiated the activity but also to neighboring units that have correlated activity. In contrast, the pontine nucleus does not have correlated activity, and the organization of its composite matrix is less obvious.

\section{ALIGNMENT OF CORTICAL VISUO-MOTOR MAPS}

In this section we describe a model of the developmental alignment of cortico-cortical connections guided by cerebellar cortical activity. Visual target information is simultaneously provided to the sensory layer and to PCs in the cerebellar cortex (Fig. 1). We assume that the connections from sensory to MC layer are initially random, whereas the cerebellar cortex has already learned to use this sensory input to guide movements to appropriate endpoints. Experimental studies of the thalamocortical projection supports a critical period during development, after which plasticity is reduced (Iriki et al. 1991; Crair and Malenka 1995; Fox et al. 1996), and on this basis we assume that the plasticity of intrinsic premotor connections is turned off after the maturation of these connections. Cortico-cortical connections, however, remain plastic throughout development and adulthood (Iriki et al. 1991). Stable premotor connections allow the cerebellum to export motor learning to cortico-cortical sites by way of reliable connections.

In our model we presented visual target inputs to the sensory cortex and cerebellar cortex concurrently. For example, if units $n-1, n$, and $n+1$ were activated at the target layer, cerebellar units $n-1, n$, and $n+1$ were simultaneously disinhibited. All other cerebellar units were continually inhibited by full PC inhibition. These concurrent inputs were presented for 75 time steps and were chosen randomly. Our implementation of concurrent target input is only a simplification of the actual pathway by which these two areas receive concurrent inputs. In reality, visual target input is relayed to the cerebellum by way of the corticopontine and the mossy fiber projection to the cerebellum (Brodal and Bjaalie 1992).

Additionally, after the first developmental stage during which the basic architecture of the premotor weights are established, the gain of the network is allowed to increase. Electrophysiologic evidence shows that immature cortical neurons are less responsive than mature units (Prince and $\mathrm{Hu}-$ guenard 1988). Additionally, an increase in gain may occur by the maturation of neuromodulatory inputs such as cholinergic or noradrenergic inputs (McCormick 1989). We model a growth in network gain by increasing the size of the normaliza-

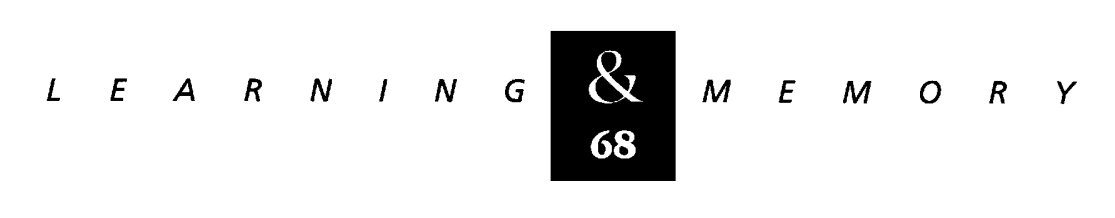


tion constant $\chi$ from 0.5 to 5.0 after the first developmental stage. This growth in weights effectively increases the gain of the input-output transformation performed by each unit, allowing activity to reverberate in the circuit.

Figure 3 shows that the feed-forward projection between the sensory cortex and the MC layer developed a continuous topographic map. Figure $3 a$ shows the initial random cortico-cortical map. After paired target inputs were given to the target layer and cerebellar layer, the cortico-cortical projection, shown in Figure 3b, developed a topographic map that corresponds closely to the cerebello-MC projection.

\section{VISUO-MOTOR LEARNING}

After the development and alignment of both the premotor circuit and the cortico-cortical projection, the network was subjected to a visual perturbation task in which the input to the visual cortex is shifted by four units. This shift in visual input is analogous to a prism adaptation task in which subjects wear prism goggles that shift visual space. A shift in visual inputs causes a shift in the pattern of PC deactivation, resulting in a movement error. We assumed that this movement error allows the cerebellum to learn the new visuo-motor mapping so that the correct movement can be made, compensating for the shift in visual target input. A previous modeling study has demonstrated that longterm depression at the parallel fiber synapse on PCs in an adjustable pattern generator (APG) model can simulate the reprogramming of movements to shifted sensory inputs (Berthier et al. 1993). In the present model, we assumed that the cerebellum has learned the new sensorimotor pro- gramming. Thus, even though visual target input is shifted at the sensory cortex, cerebellar cortical activity has learned to compensate by disinhibiting the unshifted pattern of cerebellar nuclear cells, allowing the correct movement to be initiated. Thus, shifted visual target input is paired with an unshifted disinhibition of cerebellar nuclear cells because of cerebellar learning of the new mapping. For example, if units $n-1, n$, and $n+1$ were activated at the sensory layer, cerebellar units $n+3$, $n+4$, and $n+5$ received PC disinhibition, whereas all other cerebellar units were continually inhibited by full PC inhibition. The paired inputs were presented for 75 time steps and were chosen randomly.

Figure $3 \mathrm{c}$ shows that the feed-forward projection between the sensory layer and the MC layer developed a continuous and shifted topographic map. When compared with the topographic map in the nonshifted case (Fig. 3b), the topographic map in the shifted case is appropriately shifted by four units. Thus, activation of the appropriate output by the cerebellum allows the appropriate adaptation by cortico-cortical connections.

\section{ACTIVATION DYNAMICS DURING DEVELOPMENT AND LEARNING}

So far we have discussed only the pattern of weights that arise during development and learning. However, it is important to look at the behavior of the activation dynamics that occur with each pattern of connections. The activation dynamics at three stages of development are shown. First we show the behavior of the network after the first stage when the premotor network has fully developed but when the cortico-cortical connections
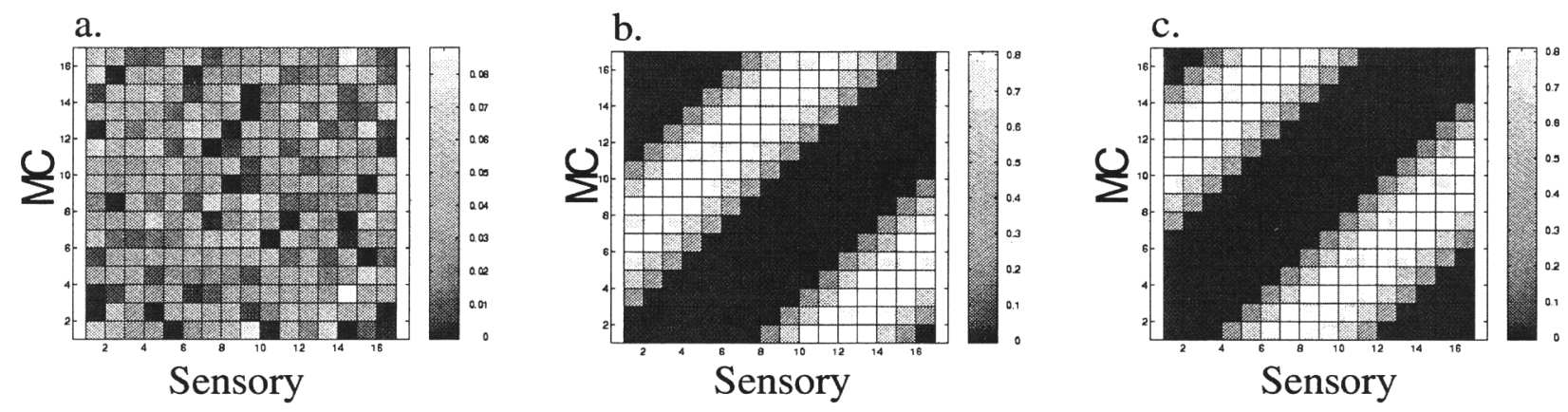

Figure 3: Development and adaptation in the cortico-cortical projection. (a) The initially random weight matrix; $(b)$ the topographically organized cortico-cortical projection formed after the second developmental stage of visuo-motor alignment; $(c)$ the shifted cortico-cortical projection after adaptation of a visuo-motor task in the learning phase.

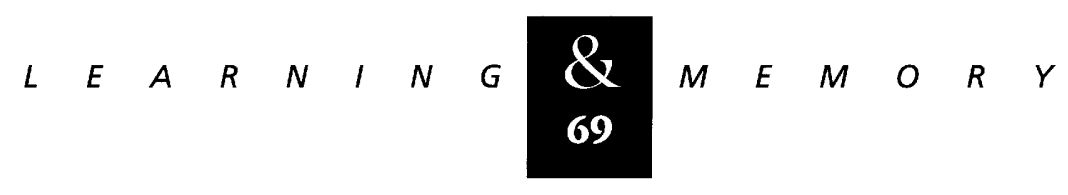


were still random, as shown in Figure 3a. Second, we show that after the second stage when the cortico-cortical connections have formed, motor activity can be initiated and driven by cortico-cortical connections alone. Finally, we show that after cerebellar learning of the visuo-motor task has been exported to the cortex, cortico-cortical connections were able to initiate motor activity in the appropriate direction to compensate for the shifted visual input.

Figure 4 shows the dynamic behavior of a network after the first developmental stage. The premotor network has formed reciprocal connections, and the weights have grown in size so that the gain of the network has increased. The projection from the sensory cortex to the MC has not yet developed and still has random connections. Figure 4 ,
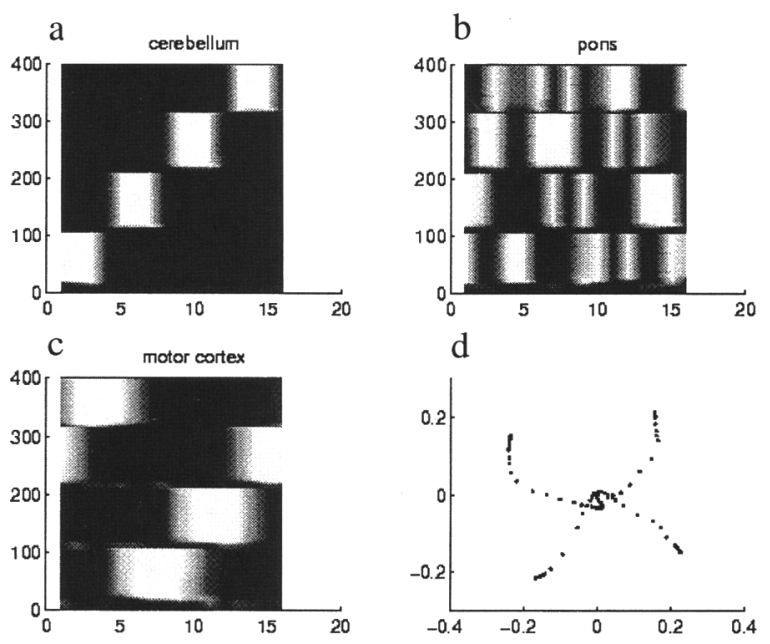

Figure 4: Activation dynamics for a network after the first developmental stage. This network has a mature premotor circuit but random cortico-cortical connections (Fig. 3a). (a-c) Activation dynamics over time of the cerebellar, pons, and MC layers when four inputs are given to the sensory cortex. Inputs are given to neighboring clusters of three units centered on units $2,7,11$, and 14. Each input is held on for 105 time steps concomitant with disinhibition of the corresponding cerebellar units. The unit number is represented on the horizontal axis, whereas the number of time steps is represented on the vertical axis. (d) Temporal evolution of the population direction vector for each of the four inputs given to the network. Each dot represents the population vector calculated at a particular time step. The population vector is calculated by a vector summation of the individual direction vectors for each unit. For each unit the amplitude of its individual vector equals its firing rate, whereas the preferred direction is calculated by the following equation (unit number/16)*2*PI. a-c, shows the activation states of the cerebellar, pontine, and MC layers, respectively, when four sets of input were sequentially presented to both the sensory cortex and the cerebellum. In these dynamic simulations, each of the four inputs was turned on for 105 time steps to fully demonstrate that the network settles to a steady state. The cerebellar layer shows four blocks of activation that correspond to the four sets of input, each causing disinhibition of a cluster of cerebellar nuclear cells. After the presentation of each new input, activity in the MC layer was initially disorganized. However, after activity had traveled through the premotor loop, the appropriate pattern of PC inhibition allowed premotor activity, and thus motor cortical activity, to be reshaped correctly.

Figure $4 \mathrm{~d}$ shows the population direction vector in cartesian coordinates for each of the four inputs. Each point represents an instantaneous population vector. Because the ends of each layer are connected, the 16 units on each layer can each represent movement in a particular direction on a two-dimensional plane spanning $360^{\circ}$. The population vector is calculated by the spatially weighted average of the activations of the 16 units on the MC layer, with the direction specifity of each unit being distributed about circular layer in $22.5^{\circ}$ intervals. Figure $4 \mathrm{~d}$ shows that the population vectors from each of the four inputs were initially disordered but became directed at the four appropriate movement directions after PC inhibition had reshaped the activity in the premotor network.

Figure 5 shows the behavior of a network after the second developmental stage when the corticocortical projection has aligned with premotor circuits. In this simulation we removed cerebellar disinhibition, such that the visual target input is only given to the sensory cortex. This arrangement simulates a network where motor learning has already been exported to cortical sites, and cerebellar control is no longer needed. Thus, the movement can be initiated directly by cortico-cortical connections. The cerebellar nuclear layer, in Figure $5 \mathrm{a}$, is silent throughout the sequential presentation of four sets of inputs. The MC layer shows four distinct blocks of activity corresponding to the correct sequence of motor activity as specified by the sensory cortical layer. The pontine layer shows clustered discontinuous activity as a result of $\mathrm{MC}$ activation. Figure $5 \mathrm{~d}$ shows the population direction vectors of the MC layer. The four distinct movement directions correspond to the four different target inputs given to the sensory cortex. Note

$$
\begin{array}{llllllllllllllll}
\hline & E & A & R & N & I & N & G & \mathbf{Q} & M & E & M & O & R & Y \\
\mathbf{7 0} & & & &
\end{array}
$$



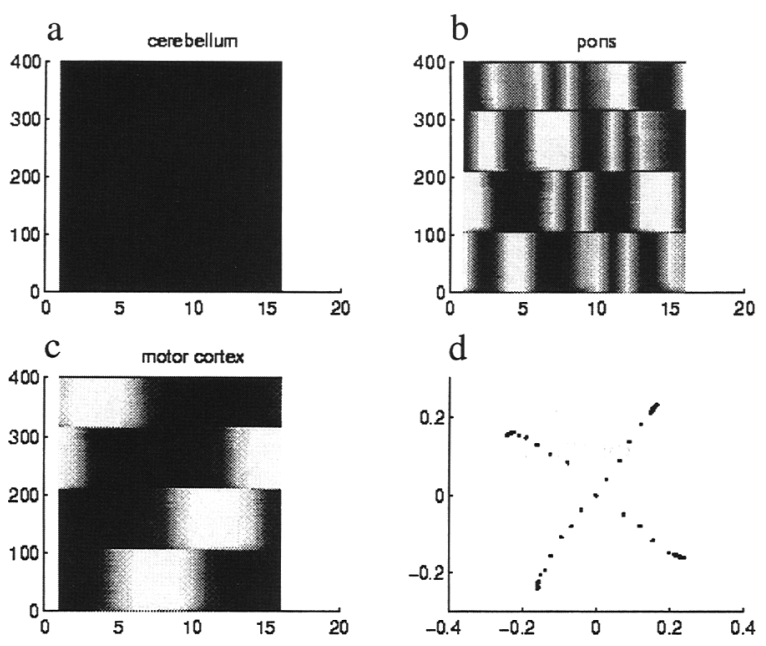

Figure 5: Activation dynamics for a network after the second developmental stage. The network has a mature premotor circuit and an aligned cortico-cortical map (Fig. 3b). All conventions are the same as that in Fig. 4, except no cerebellar disinhibition occurs in this simulation.

that with each input, the population vector initially grows in amplitude, and then the population vector remains fixed at a particular direction and amplitude.

Figures 6 and 7 show the behavior of a network that is exposed to a visuo-motor adaptation task. Figure 6 shows the activation dynamics after the cerebellum has learned to compensate for the 4-unit shift in visual target space but before exportation of the learning to cortical sites. Because the cortico-cortical connections have not adapted to the shift in target space, visual inputs to the sensory layer initially activate inappropriate motor units in the MC by direct cortico-cortical connections. However, because the cerebellum has learned to compensate for visual shift, the $\mathrm{MC}$ is able to attain the correct pattern of activation by PC shaping of premotor activity, shown in Figure 6c. Figure $6 \mathrm{~d}$ shows the shift or rotation of the direction vector that occurs when cerebellar inhibition guides the behavior of MC activity.

After cerebellar learning of the visuo-motor task has been exported to the cortex, the corticocortical projection directs the appropriate pattern of MC activity in response to the shifted target inputs. Figure 7 shows a simulation where exportation of learning has already taken place, and the cerebellum no longer responds to the visual input. Figure 7 a shows that the cerebellar nucleus has no activity. Instead, the sensory cortex is able to di- rectly activate the correct pattern of $\mathrm{MC}$ units to compensate for the shift in visual target space. Figure $7 \mathrm{~d}$ shows that the population direction vector grows directly in the correct direction through the realigned cortico-cortical connections.

\section{Discussion}

These modeling studies demonstrate that cerebellar output could be used to guide the development of premotor circuits as well as to direct sensorimotor learning in cortico-cortical connections. Spontaneous PC disinhibition with rebound activation of cerebellar nuclear units allowed the formation of reciprocal connections in the premotor network. These reciprocal connections permit activity to persist and reverberate in specific computational modules. After development of the premotor network is completed, paired target information to a visual sensory area of cortex and to the cerebellar cortex allowed the alignment of cortico-cortical maps. When visual target information is misaligned with movement, mimicking prism adaptation, cerebellar learning of the misalignment directs realignment of cortico-cortical connections. After realign-
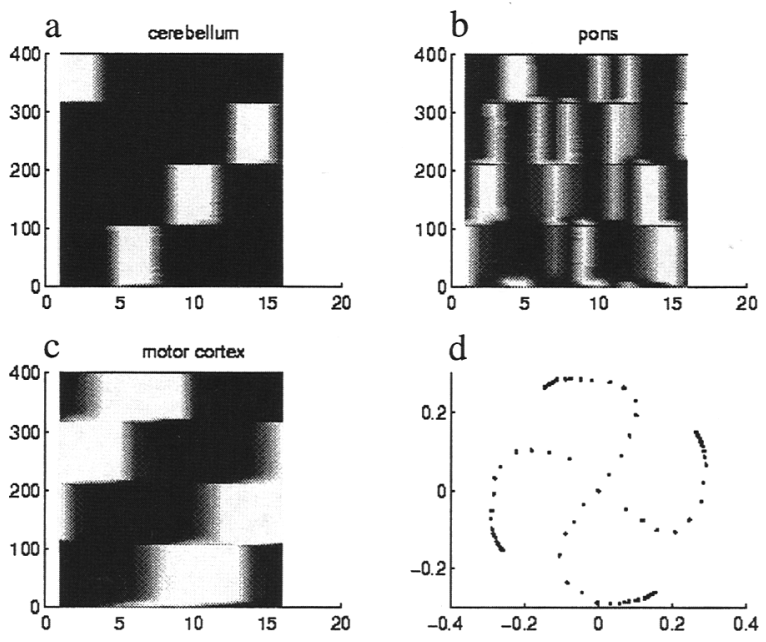

Figure 6: Activation dynamics for a network after the second developmental stage but subjected to a visuomotor task. The network has a mature premotor circuit and an aligned cortico-cortical map (Fig. 3b). Inputs to the sensory cortex are shifted four units from the correct target. We assumed that the cerebellum has learned this visuo-motor mapping and disinhibits nuclear cells that are shifted by four units from the inputs given to sensory cortex. All other conventions are the same as that in Fig. 4.

$$
\begin{array}{llllllllllllllll}
L & E & A & R & N & I & N & G & \begin{array}{l}
\boldsymbol{Z} \\
\mathbf{7 1}
\end{array} & M & E & M & O & R & Y
\end{array}
$$



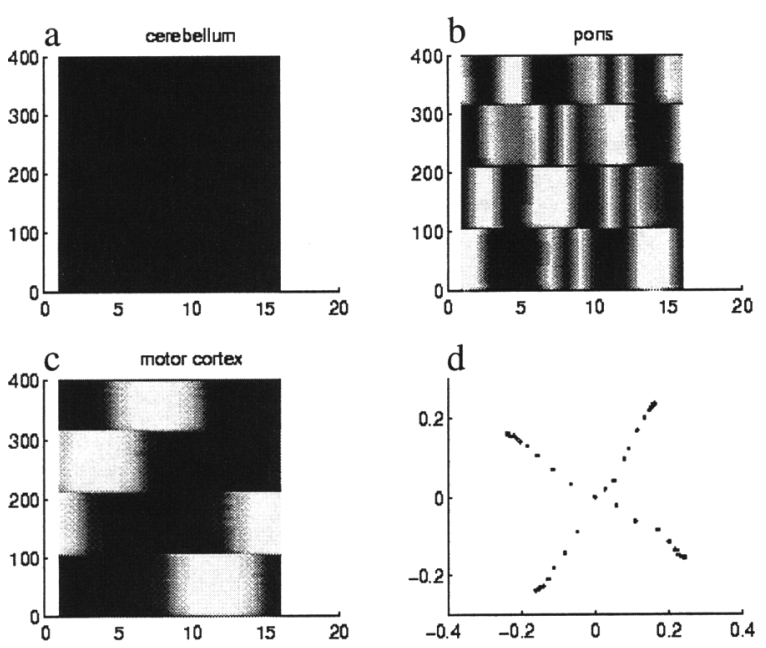

Figure 7: Activation dynamics for a network that has learned the visuo-motor task. The premotor circuit remains unchanged, but the cortico-cortical map is shifted as shown in Fig. 3c. Inputs to the sensory cortex are shifted by four units from the correct target. A corresponding shift in cortico-cortical connections alone, without disinhibition in the cerebellum, can compensate for the shift in visual inputs. All other conventions are the same as that in Fig. 4.

ment of cortical connections is accomplished, cortical areas supplying visual target information are able to initiate movement in the appropriate direction to move toward a target without further involvement of the cerebellum. Elimination of cerebellar involvement is advantageous, as it shortens the control pathway for responses that are used frequently, thus forming what has been referred to as "sensorimotor habits" (Houk and Barto 1992).

\section{MODELING ASSUMPTIONS}

Synaptic plasticity simulated in the present model resulted from Hebbian learning coupled with weight normalization. Hebbian learning is thought to be implemented by the NMDA channel (Brown et al. 1990) and has been demonstrated in several regions of the CNS including the cerebral cortex (Bear and Kirkwood 1993). Labeling studies have demonstrated NMDA labeling in cortex, thalamus, pons, and cerebellar nuclei (Petralia et al. 1994). These studies suggest that Hebbian learning could occur at each of the layers in the present model.

Labeling studies have shown that NMDA receptor subunits have differential expression pat- terns during development (Zhong et al. 1995). Thus, different levels of plasticity could occur during development. In the present model we assumed that plasticity in the premotor network occurred during early development. Several experimental studies show that the thalamo-cortical connection has a critical period for synaptic plasticity after which plasticity is reduced (Fox et al. 1996). Such a critical period has been demonstrated for several sensory cortical areas (Fox and Zahs 1994). In addition, Iriki et al. (1991) have shown that adult thalamo-cortical connections to motor cortex are less plastic when compared with cortico-cortical connections to the motor cortex. Although these studies strongly suggest that thalamo-cortical synapses become less plastic after early development when compared with corticocortical synapses, little is known about synaptic plasticity at other synapses in the premotor circuit. Further studies are needed to confirm the assumption that premotor synapses become less plastic after early development.

Hebbian learning is generally unstable because it only specifies a weight increment. Most models of recurrent networks include a weight decay mechanism to limit learning (Shinomoto 1987). In the present model, we used dual weight normalization to stabilize synaptic learning. Forms of weight normalization exist biologically at the level of nuclei (Hayes and Meyer 1988a,b) and at the level of neurons (Markram and Tsodyks 1996). Furthermore, most models of Hebbian learning in feed-forward networks utilize weight normalization (Whitelaw and Cowan 1981; Miller et al. 1989; Miller 1994). Such models can simulate the development of feed-forward pathways such as topographic maps, ocular dominance columns, and orientation domains. We have shown recently that weight normalization with Hebbian learning is effective in recurrent networks (S.E. Hua, F.A. MussaIvaldi, and J.C. Houk, unpubl.). In that study weight normalization in a two-layered recurrent network allowed the formation of symmetric, reciprocal, and modular connections.

During the transition from the first to the second developmental phases, we assumed that synaptic weights in the premotor network grew in strength. This growth allows the mature premotor circuit to have sufficient network gain to sustain reverberatory activity. This up-regulation in the strength of synapses was accomplished by an increase in the normalization constant. There are two mechanisms that could accomplish such a

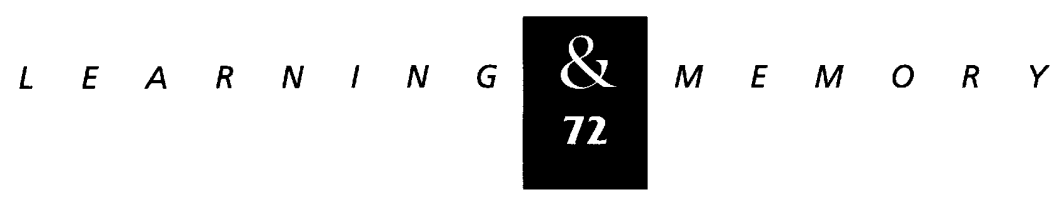


transition in weight. First, during development, weights could undergo a gradual increase in the size of connections or neuronal responsiveness as neurons mature. Immature cortical neurons have been found to be less responsive than adult neurons (Prince and Huguenard 1988). Second, an increase in responsiveness can be achieved by modulatory neural transmitters. Cholinergic and adrenergic input have been shown to have both inhibitory and excitatory effects on cortical excitability (McCormick 1989). Both of these mechanisms could exist to allow an increased network gain after premotor connections are formed.

During the late developmental and learning phases, visual target information is provided simultaneously to the sensory cortical layer and to PCs. During the prism adaptation task, rotated target information is again provided to both layers, but the PCs are assumed to have learned the correct counter-rotation to achieve the appropriate movement. For simplicity, we did not explicitly include cerebellar learning in this model. Instead, we assumed that the cerebellum would be able to learn this counter-rotation to achieve the proper movement in spite of rotated target information. Previously, we have shown that an APG model of cerebellar function can learn the alignment between target and movement direction as well as adapt to visual distortion (Berthier et al. 1993). This model is consistent with the Marr-Albus theory of parallel fiber-PC synapse modification by error signals from climbing fibers (Marr 1969; Albus 1971). In the present model, learning at the cerebellar cortex is then transmitted through premotor networks to modify cortico-cortical connections.

Of the modeling assumptions presented above, the architecture of the network, Hebbian learning with weight normalization, and developmental windows of plasticity are assumptions with a fair amount of experimental support. The assumption involving growth in network gain after premotor circuits have developed is more speculative. We have shown previously that in recurrent networks that learn, low network gain allows the formation of modules that provide a more accurate representation of the set of inputs (S.E. Hua, F.A. Mussa-Ivaldi, and J.C. Houk, in prep.). If network gain is high during learning, reciprocal and modular connections still develop, but internal feedback overshadows the contribution to loop activity by external inputs. However in the present model, PC inhibition may be able to control high levels of feedback activity in the recurrent network.
We propose two major predictions of the model. First, the development of reciprocal and modular connections is a robust feature of the model. Thus, the model predicts that anatomical labeling studies should find results consistent with reciprocal and modular connectivity. We propose that simultaneous labeling of a single motor cortical cell by a retrograde and anterograde viral tracer would allow double labeling of cells involved in the same module in other layers of the network. The second prediction involves electrophysiological evidence for the rotation of the population vector after exportation is complete and is presented in a later section on computational modules.

\section{TOPOGRAPHIC ALIGNMENT OF THE NETWORK}

Although we describe the topographic organization of the weights in this study, the weights may be described more accurately in terms of functional topology. Because the layers are of one dimension and wrapped, it is difficult to directly apply the topography of these layers to the threedimensional brain. More important, the topological organization of the connections come from the correlation structure of the inputs and of the network activity. We allowed the inputs (cortical and PC) to be topographically correlated so that the organization of the connections would be easier to visualize. However, such topographic correlations need not exist. Instead, the correlations used in this model belong to a more general class of topologic or functional correlations. Neighboring units on each layer do not necessarily represent spatial neighbors; instead, neighboring units are functional neighbors. Correlated PC activity represents correlations of directional tuning or muscle synergies and not necessarily spatial topographic relationships. Thus, the actual topographic layout of modularity in the three-dimensional brain is difficult to predict from this study, because the results predict mainly functional topology. Additionally, simple functional topologic relationships such as orientation tuning have complex two-dimensional topography in the visual cortex.

\section{COMPUTATIONAL MODULES FOR ELEMENTAL MOTOR COMMANDS}

The present results illustrate that reciprocity is a natural outcome of Hebbian learning in recurrent

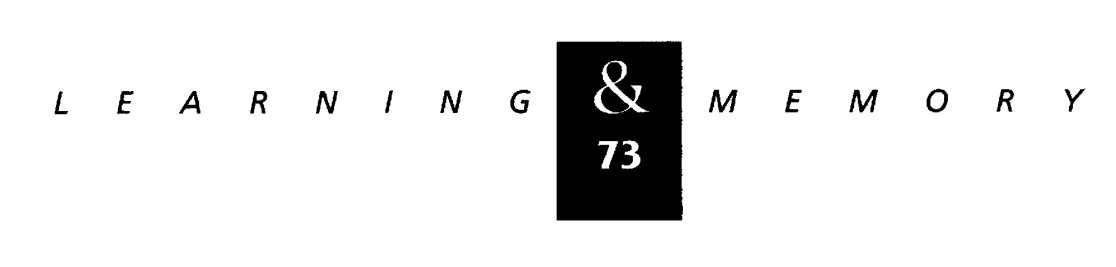


networks. Strict reciprocity exists when activity initiated in one unit will only return to that one unit without divergence to other units on that layer. In contrast, the present simulation results show that reciprocal connections with divergent recurrent connections develop in the premotor circuit. The divergent connections are made between units having correlated activity such as neighboring cortical units with lateral connections or neighboring cerebellar nuclear units with common PC disinhibition. Whereas reciprocal connections sustain activity in a particular premotor circuit, divergent recurrent connections allow recruitment of premotor circuits with similar functional properties, that is, correlated activity. Highly correlated premotor circuits with strong, divergent interconnections are considered as computational modules that cooperate to produce an elemental motor command (Houk et al. 1993). Weaker divergent connections between different modules serve to combine elemental motor commands under the guidance of the cerebellar cortex.

In the present model, divergent connections allow the population direction vector to rotate from a shifted visual target to the corrected movement direction derived from cerebellar learning (see also Eisenman et al. 1991). Although the visuomotor task simulated in the present model was prism learning, the present results provide similar population behavior as that seen in the rotation of the population direction vector in monkey motor cortex (Lurito et al. 1991). In these experiments, monkeys made arm movements (hand-held manipulandum) to one of eight equally spaced target lights placed in a circle around the starting position on a two-dimensional surface. Interspersed with direct arm movements to dimly lit targets, monkeys were trained to make arm movements $90^{\circ}$ counterclockwise (CCW) to a bright target light. Motor cortical single unit recording showed that the population direction vector initially points toward the nonrotated target light and subsequently rotates to the $90^{\circ} \mathrm{CCW}$ target.

Rotation of the population vector similar to the experiment of Lurito et al. (1991) is seen in the present model when the cerebellum has learned the new visuo-motor mapping but this learning has not yet been exported to the motor cortex (Fig. 6). After cerebellar learning has been exported to the cortex, the population vector no longer rotates because cortico-cortical connections have learned to initiate movement in the correct direction (Fig. 7). These results predict that if monkeys were trained on only the rotated arm movements, rotation of the population vector would diminish as cerebellar learning is exported to cortical sites. Our results further suggest that the presence of the rotating population vector in monkey experiments may occur at a stage where the cerebellum has learned the correct visuo-motor mapping, but this learning has not been exported to the cortex. It is possible that the intermixing of nonrotated and rotated movements in the same block may have slowed the exportation of the rotated visuo-motor task.

The present model demonstrates that the initiation of movements in the appropriate direction can be exported from the cerebellum to cortical sites. This model is a first step in describing the exportation of both movement initiation and execution to motor cortex. We have shown previously that the APG model of cerebellar function programs movement execution by a dynamic pattern of PC activation. In this way, movements are terminated by the activation of PCs. The exportation of movement execution and termination to cortical networks remains a difficult problem and will be approached in future modeling efforts.

\section{Acknowledgments}

We thank Ferdinando Mussa-Ivaldi and Andrew Barto for helpful discussions. This work was supported by National Institutes of Health/National Institute of Mental Health grant 5-P50-MH48185 to J.C.H.

The publication costs of this article were defrayed in part by payment of page charges. This article must therefore be hereby marked "advertisement" in accordance with 18 USC section 1734 solely to indicate this fact.

\section{References}

Albus, J.S. 1971. A theory of cerebellar function. Math. Biosci. 10: 25-61.

Anderson, J.A., J.W. Silverstein, S.A. Ritz, and R.S. Jones. 1977. Distinctive features, categorical perception, and probability learning: Some applications of a neural model. Psychol. Rev. 84: 413-451.

Bauer, J. and R. Held. 1971. Comparison of visually guided reaching in normal and deprived infant monkeys. J. Exp. Psychol. Anim. Behav. Processes 1: 298-308.

Bear, M.F. and A. Kirkwood. 1993. Neocortical long-term potentiation. Curr. Opin. Neurobiol. 3: 197-202.

Berthier, N.E., S.P. Singh, A.G. Barto, and J.C. Houk. 1993. Distributed representation of limb motor programs in arrays of adjustable pattern generators. J. Cognit. Neurosci. 5: $56-78$.

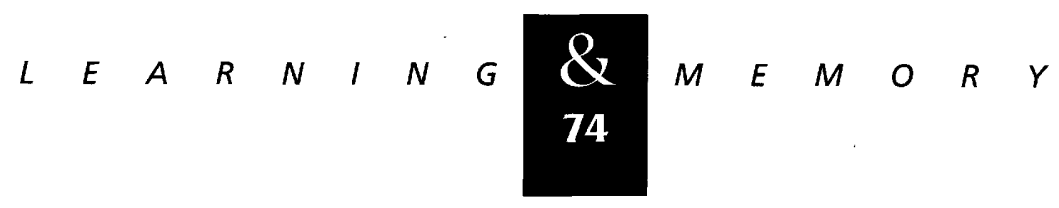


Bloedel, J.R., T.J. Ebner, and S.P. Wise. 1996. Acquisition of motor behavior in vertebrates. MIT Press, Cambridge, MA.

Brodal, P. and J.G. Bjaalie. 1992. Organization of the pontine nuclei. Neurosci. Res. 13: 83-118.

Brown, T.H., E.W. Kairiss, and C.L. Keenan. 1990. Hebbian synapses: Biophysical mechanisms and algorithms. Annu. Rev. Neurosci. 13: 475-511.

Crair, M.C. and R.C. Malenka. 1995. A critical period of long-term potentiation at thalamocortical synapses. Nature 375: 325-328.

Ebner, T.J., D. Flament, and S.J. Shanbhag. 1996. The cerebellum's role in voluntary motor learning: Clinical, electrophysiological, and imaging studies. In Acquisition of motor behavior in vertebrates (ed. J.R. Bloedel, T.J. Ebner, and S.P. Wise), pp. 235-260. MIT Press, Cambridge, MA.

Eisenman, L.N., J. Keifer, and J.C. Houk. 1991. Positive feedback in the cerebro-cerebellar recurrent network may explain rotation of population vectors. In Analysis and modeling of neural systems (ed. F. Eeckman), pp. 371-376. Kluwer Academic Publishers, Norwell, MA.

Fox, K. and K. Zahs. 1994. Critical period control in sensory cortex. Curr. Opin. Neurobiol. 4: 112-119.

Fox, K., B.L. Schlaggar, S. Glazewski, and D.D.M. O'Leary. 1996. Glutamate receptor blockade at cortical synapses disrupts development of thalamocortical and columnar organization in somatosensory cortex. Proc. Natl. Acad. Sci. 93: 5584-5589.

Friston, K.J., C.D. Frith, R.E. Passingham, P.F. Liddle, and R.S. Frackowiak. 1992. Motor practice and neurophysiological adaptation in the cerebellum: A positron tomography study. Proc. R. Soc. Lond. - Ser. B: Biol. Sci. 248: 223-228.

Galiana, H.L. 1986. A new approach to understanding adaptive visual-vestibular interactions in the central nervous system. J. Neurophysiol. 55: 349-374.

Ghosh, S., G.M. Murray, A.B. Turman, and M.J. Rowe. 1994. Corticothalamic influences on transmission of tactile information in the ventro-posterolateral thalamus of the cat: Effect of reversible inactivation of somatosensory cortical areas I and II. Exp. Brain Res. 100: 276-286.

Gilbert, P.F.C. 1974. A theory of memory that explains the function and structure of the cerebellum. Brain Res. 70: 1-18.

Hayes, W.P. and R.L. Meyer. 1988a. Optic synapse number but not density is maintained during regeneration onto surgically-halved tectum in goldfish: HRP-EM evidence that optic fibers compete for fixed numbers of postsynaptic sites on tectum. J. Comp. Neurol. 274: 539-559.

1988b. Retinotopically inappropriate synapses of subnormal density formed by surgically misdirected optic fibers in goldfish tectum. Brain Res. 38: 304-312.
Houk, J.C. 1997. On the role of the cerebellum and basal ganglia in cognitive signal processing. Prog. Brain Res. 114: $545-554$.

Houk, J.C. and A.G. Barto. 1992. Distributed sensorimotor learning. In Tutorials in motor behavior II (ed. G.E. Stelmach and J. Requin), pp. 71-100. Elsevier, Amsterdam, The Netherlands.

Houk, J.C., S.P. Singh, C. Fisher, and A.G. Barto. 1990. An adaptive sensorimotor network inspired by the anatomy and physiology of the cerebellum. In Neural networks for control (ed. W.T. Miller, R.S. Sutton, and P.J. Werbos), Chapter 13, pp. 301-348. MIT Press, Cambridge, MA.

Houk, J.C., J. Keifer, and A.G. Barto. 1993. Distributed motor commands in the limb premotor network. Trends Neurosci. 16:27-33.

Iriki, A., C. Pavlides, A. Keller, and H. Asanuma. 1991. Long term potentiation of thalamic input to the motor cortex induced by coactivation of thalamocortical and corticocortical afferents. J. Neurophysiol. 65: 1435-1441.

Ito, M. 1989. Long-term depression. Annu. Rev. Neurosci. 12: $85-102$.

Keifer, J. 1996. Effects of red nucleus inactivation on burst discharge in turtle cerebellum: Evidence for positive feedback. J. Neurophysiol. 76: 2200-2210.

Keifer, J., D. Vyas, and J.C. Houk. 1992. Sulforhodamine labeling of neural circuits engaged in motor pattern generation in the in vitro turtle brainstem-cerebellum. J. Neurosci. 12: 3187-3199.

Kim, S.-G., K. Ugurbil, and P.L. Strick. 1994. Activation of a cerebellar output nucleus during cognitive processing. Science 265: 949-951.

Lurito, J.T., T. Georgakopoulos, and A.P. Georgopoulos. 1991. Cognitive spatial-motor processes 7 . The making of movements at an angle from a stimulus direction: studies of motor cortical activity at the single cell and population levels. Exp. Brain Res. 87: 562-580.

Ma, W. and S.L. Juliano. 1991. The relationship between thalamocortical connections and stimulus-evoked metabolic activity in the ventroposterior nucleus of the monkey. Somatosens Mot. Res. 8: 77-86.

Markram, H. and M. Tsodyks. 1996. Redistribution of synaptic efficacy between neocortical pyramidal neurons. Nature 382: 807-810.

Marr, D. 1969. A theory of cerebellar cortex. J. Physol. (Lond.) 202: 437-470.

McCormick, D.A. 1989. Cholinergic and noradrenergic modulation of thalamocortical processing. Trends Neurosci. 12: $215-221$.

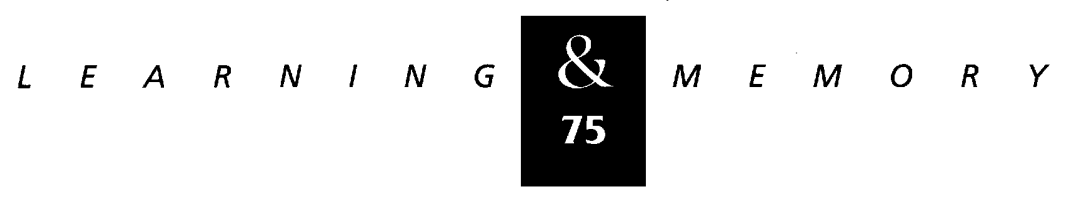


Milak, M.S., V. Bracha, and J.R. Bloedel. 1995. Relationship of simultaneously recorded cerebellar nuclear neuron discharge to the acquisition of a complex, operantly conditioned forelimb movement in cats. Exp. Brain Res. 105: 325-330.

Miller, K.D. 1994. A model for the development of simple cell receptive fields and the ordered arrangement of orientation columns through activity-dependent competition between ON- and OFF-center inputs. J. Neurosci.

14: $409-441$.

Miller, K.D. and D.J.C. MacKay. 1994. The role of constraints in Hebbian learning. Neural Comput. 6: 100-126.

Miller, K.D., J.B. Keller, and M.P. Stryker. 1989. Ocular dominance column development: Analysis and simulation. Science 245: 605-615.

Petralia, R.S., N. Yokotani, and R.J. Wenthold. 1994. Light and electron microscope distribution of the NMDA receptor subunit NMDAR1 in the rat nervous system using a selective anti-peptide antibody. J. Neurosci. 14: 667-696.

Prince, D.A. and J.R. Huguenard. 1988. Functional properties of neocortical neurons. In Neurobiology of neocortex (ed. P. Rakicand and W. Singer), pp. 153-176. John Wiley \& Sons, Chichester, UK.

Raichle, M.E., J.A. Fiez, T.O. Videen, A.K. Macleod, J.V. Pardo, P.T. Fox, and S.E. Petersen. 1994. Practice-related changes in human brain functional anatomy during nonmotor learning. Cereb. Cortex 4: 8-26.

Raymond, J.L., S.G. Lisberger, and M.D. Mauk. 1996. The cerebellum: A neuronal learning machine? Science 272: $1126-1131$.

Shinomoto, S. 1987. Memory maintenance in neural networks. J. Phys. A: Math. Gen. 20: L1305-L1309.

Thach, W.T., H.P. Goodkin, and J.G. Keating. 1992.

Cerebellum and the adaptive coordination of movement. Annu. Rev. Neurosci. 15: 403-442.

Thompson, R.F. 1986. The neurobiology of learning and memory. Science 233: 941-947.

Tsukahara, N., N. Bando, T. Murakami, and Y. Oda. 1983. Properties of cerebello-precerebellar reverberating circuits. Brain Res. 274: 249-259.

Tyrrell, T. and D. Willshaw. 1992. Cerebellar cortex: Its simulation and the relevance of Marr's theory. Philos. Trans. R. Soc. Lond. - Ser. B: Biol. Sci. 336: 239-257.

Whitelaw, V.A. and J.D. Cowan. 1981. Specificity and plasticity of retinotectal connections: A computational model. J. Neurosci. 1: 1369-1387.

Zhong, J., D.P. Carrozza, K. Williams, D.B. Pritchett, and P.B. Molinoff. 1995. Expression of mRNAs encoding subunits of the NMDA receptor in developing rat brain. J. Neurochem. 64: $531-539$.

Received February 24, 1997; accepted in revised form May 7, 1997. 


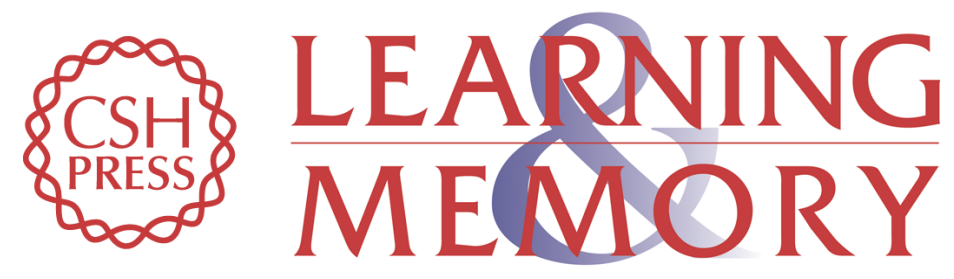

\section{Cerebellar guidance of premotor network development and sensorimotor learning.}

S E Hua and J C Houk

Learn. Mem. 1997, 4:

Access the most recent version at doi:10.1101/lm.4.1.63

References This article cites 42 articles, 9 of which can be accessed free at: http://learnmem.cshlp.org/content/4/1/63.full.html\#ref-list-1

License

Email Alerting Receive free email alerts when new articles cite this article - sign up in the box at the Service top right corner of the article or click here. 